\title{
Electrical Engineering 2020 with Gill's electronic theory of magnetism 1964
}

\author{
Avtar Singh Gill
}

avtargillmd@aol.com

\begin{abstract}
It will be explained why Gill's electronic theory of magnetism 1964 should replace Maxwell's dipole theory of magnetism 1873 and its equations.

The following issues related to Maxwell's equations will be re-visited and explained with the help of Gill's electronic theory of magnetism 1964:

1. Magnetic Gauss' law to be replaced with Gill's electronic theory of magnetism 1964.

2. Electric Gauss' law will be modified.

3. Ampere's law and Ampere's right-hand rule to be combined with Gill's electronic theory of magnetism 1964.

4. Faraday's law of induction ${ }^{2}$ already explained with the help of Gill's electronic theory of magnetism 1964.
\end{abstract}

Gill's electronic theory of magnetism 1964 shows that the magnetic force has its longitudinal and its positive and negative torque components and how the two forces from the two ends of a magnet entwine to manifest as a single magnetic force $\boldsymbol{B}$.

The electrical force $\mathbf{E}$ will be shown to be a combination of the electron (-e) based negative force and the proton (+e) based positive force.

The working of a capacitor is explained and brings us closer to understanding that the electrical force and the magnetic force are both combinations of the negative electron (-e) dependant and positive proton (+e) dependant forces.

How a copper wire in the center of an electric coil can develop an increased electron (-e) based electric current which is also rotating around the central copper wire (Coil effect) ${ }^{n}$ will be explained and calculated and should be used as a renewable energy source.

Application of Gill's electronic theory of magnetism 1964 explains the working of a cyclotron and an electric motor.

In the end, it will also be shown that the travelling force as a wave is not a combination of the electrical force and the electromagnetic force but a combination of the negative electron dependant force and the positive proton dependant force as they entwine while heading for each other.

Electrical Engineering 2020 with Gill's electronic theory of magnetism 1964

\section{INTRODUCTION:}

Electrical forces and Magnetism are the pillars of Electrical Engineering. A direct relationship between electrical forces and magnetism has been long recognized and is better explained by moving away from Maxwell's dipole theory of magnetism 1873 towards 'Gill's electronic theory of magnetism 1964' 1 .

It is important to recognize natures ability to merge the proton (+e) dependant positive forces and the electron (-e) dependant negative forces and make it look like a single force.

This allows us to recognize the similarity between

B the magnetic force (a combination of two forces from its positive proton dependant (+e) south magnetic pole $\mathrm{S}$ and the negative electron dependant (-e) north magnetic pole N) and

E the electrical force (a combination of the positive proton dependant (+e) and the negative electron dependant (-e) forces).

The Magnetic force $\mathbf{B}$ and the Electrical force $\mathbf{E}$ are both combinations of electron (-e) based negative and proton $(+\mathrm{e})$ based positive forces and that is why one could give rise to the other and the asymmetry issue between electrical and magnetic forces goes away with the application of Gill's electronic theory of shows that the magnetic force is a combination of the exposed inner magnetism 1964.

Thus, the travelling force is a combination of the proton $(+\mathrm{e})$ dependant positive force and the electron (-e) dependant negative force and not a combination of the electromagnetic force and the electrical force as the latter two are two force combinations themselves.

It is important to set aside Maxwell's dipole theory of magnetism 1873 and the related Maxwell's equations for Gill's electronic theory of magnetism 1964 and the associated explanations.

Dot products explain the presence and the calculations of the forces and there is no need for the cross-product equations of Henrik Lorentz 1893.The magnetic force as defined in Teslas becomes easily convertible to a Coulomb based force or vice versa with the application of Gill's electronic theory of magnetism 1964.

The working of a cyclotron, an electric motor, a capacitor and the $(\text { Coil effect })^{\mathrm{n}}$ for multiplication of milder forces which are everywhere will be presented with the help of 'Gill's electronic theory of magnetism 1964'.

\section{METHOD}

'Maxwell's dipole theory of magnetism 1873' will be summarized followed by 'Gill's electronic theory of magnetism 1964' and the latter 
electron (-e) based negative force with a negative torque $(-\tau)$ as the north magnetic pole $\mathrm{N}$ and the exposed proton $(+\mathrm{e})$ based positive force with a positive torque $(+\tau)$ as the south magnetic pole $\mathrm{S}$ from the two magnetic ends.

It will be shown diagrammatically how the two forces from the exposed inner electron (-e) based negative force and the exposed proton $(+e)$ based positive force from the two ends of a magnet entwine as they head for each other to appear as a single force.

Likewise, the electrical force is also a combination of the negative force from the free valence electrons (-e) and the positive force from the exposed protons $(+\mathrm{e})$ called 'holes' in electrical engineering which become available when the free valence electrons flow or move along.

The electron (-e) based and the proton $(+\mathrm{e})$ based forces head for each other and manifest as the entwined electrical force from an electric wire.

As the magnetic force and the electrical force are both a combination of two forces, it is incorrect to treat the travelling force as a combination of the electromagnetic force and the electrical force.

The calculation between the magnetic force in Teslas and the electrical force in Newtons will be presented with the help of Gill's electronic theory of magnetism $1964^{3}$.

The multiplication of an electric current in the central copper wire inside an electric coil (Coil effect $)^{\mathrm{n}}$ will be calculated and presented.

The cyclotron functioning and the electric motor functioning will be presented with the help of Gill's electronic theory of magnetism 1964 and the Ampere's right-hand rule.

The capacitor functioning will be presented to show that when charged, it has electron (-e) and proton (+e) based forces entwine with each other.

Gill's electronic theory of magnetism 1964 leads to dot product calculations without any need for the cross-product calculations of Henrik Lorentz 1893.

\section{Gill's electronic theory of Magnetism 1964 versus Maxwell's dipole theory 1873}

Before discussing Gill's electronic theory of magnetism 1964, a few words about Maxwell's dipole theory of magnetism $1873^{4}$ and why it should be replaced.

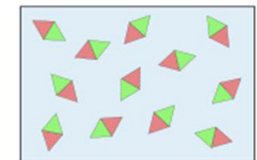

Fig 1a jumbled latice of tiny dipole magnets $\uparrow \uparrow \uparrow \uparrow \uparrow \uparrow \uparrow \uparrow$

$\uparrow \uparrow \uparrow \uparrow \uparrow \uparrow \uparrow \uparrow \uparrow$

$\uparrow \uparrow \uparrow \uparrow \uparrow \uparrow \uparrow \uparrow$

Fig 1 b tiny dipole magnets have straightened out
Maxwell's Theory of Electro-Magnetism 1873 as shown in Fig 1a above describes ferromagnetic metals consisting of tiny dipole magnets (every north pole is yoked to a south pole) and in a non-magnetic state does not manifest any free north-pole and south-pole as they are a jumbled lattice and in Fig $1 \mathrm{~b}$ straighten out during magnetization into north to south direction when magnetized. From Fig 1a and Fig 1b, the magnetic force appears to be a single force. This leads to the asymmetry issue.

Gill's electronic theory of magnetism 1964 is based on the structure of the atom and explains how the positively charged protons $(+e)$ and the negatively charged electrons (-e) are responsible for both magnetism and electrical forces. The asymmetry issue also gets resolved.

In the diagrams that follow in this article, we are using a simplified version of the structure of an atom with a large black proton mass and small red electrons.

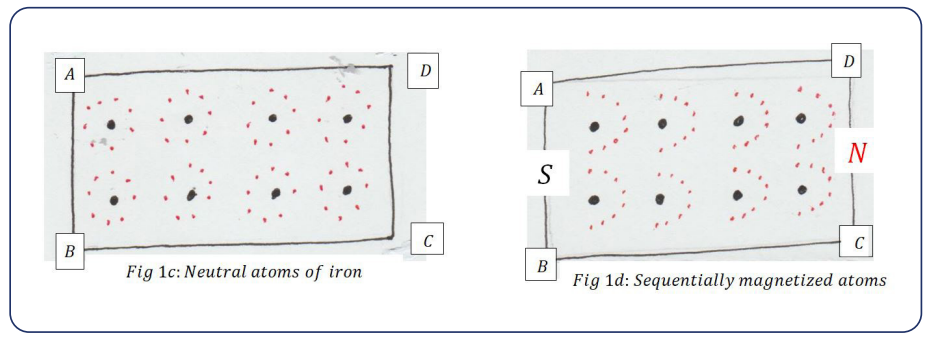

Gill's electronic theory of magnetism 1964 shows the neutral iron atoms in Fig 1c are magnetized sequentially in Figure 1d and CD has become the negative or north magnetic pole $\mathrm{N}$ with exposed inner electrons $(-\mathrm{e})$ with a negative torque $(-\tau)$ manifesting as a non-moving charge - $\boldsymbol{n} \boldsymbol{e}$ and $\mathrm{AB}$ has become the positive or the south magnetic pole $\mathrm{S}$ of the magnet with exposed protons $(+\mathrm{e})$ with an oppositely positively torqued $(+\tau)$ non-moving charge $+\boldsymbol{n} \boldsymbol{e}$ where $\boldsymbol{n}$ is the number of exposed inner electrons at one end and equals the number of exposed protons at the other end.

Neutral atoms in Fig 1c have become magnetized atoms in Fig 1d by undergoing a change in configuration and each magnetized atom has also developed an opposing torque between its own exposed inner electrons and protons.

Figure 1d shows that the magnetic force comprises of two forces emanating from the proton (+e) dependant south magnetic pole $\mathrm{S}$ and the electron (-e) dependant north magnetic pole $\mathrm{N}$ of reconfigured magnetized atoms.

$$
\text { Why }(+e) \text { and }(-e) \text { forces spin }
$$

Applying Gill's electronic theory of magnetism 1964 to the following electro-magnetic experiment shows how and why the two forces spin and entwine while they head for the opposite source. 


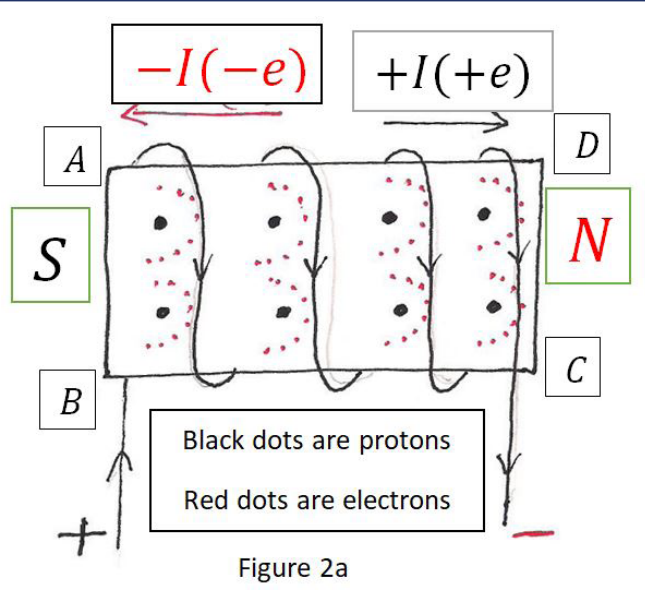

Gill's electronic theory of magnetism 1964 has been applied to a piece of iron as it is magnetized by a direct electric current coil as shown in Figure 2a.

The negative free valence electrons (-e) as they flow as a negative electric current in the coil in the negative direction towards $\mathrm{AB}$ act on the iron piece inside the coil and lead to an exposure of protons by attraction at the south magnetic pole $\mathrm{S}$ and these exposed protons at the magnetic end have also developed a positive torque $(+\tau)$ by attraction in the direction of the circular electron flow in the electro-magnetic coil.

The same negative free valence electron (-e) flow in the electric coil causes the exposed inner electrons of atoms in the iron piece inside the electric coil to be repelled sequentially towards CD to become the north magnetic pole $\mathrm{N}$ and these exposed inner electrons have developed a negative torque $(-\tau)$ by repulsion from the circular electron flow in the electro-magnetic coil.

The atoms in the iron piece are getting sequentially magnetized and at every inter-atomic intra-magnetic level there is an exposed proton (+e) based south magnetic pole $\mathrm{S}$ with a positive torque $(+\tau)$ facing an exposed inner electron $(-\mathrm{e})$ based north magnetic pole $\mathrm{N}$ with a negative torque $(-\tau)$ of the next atom.

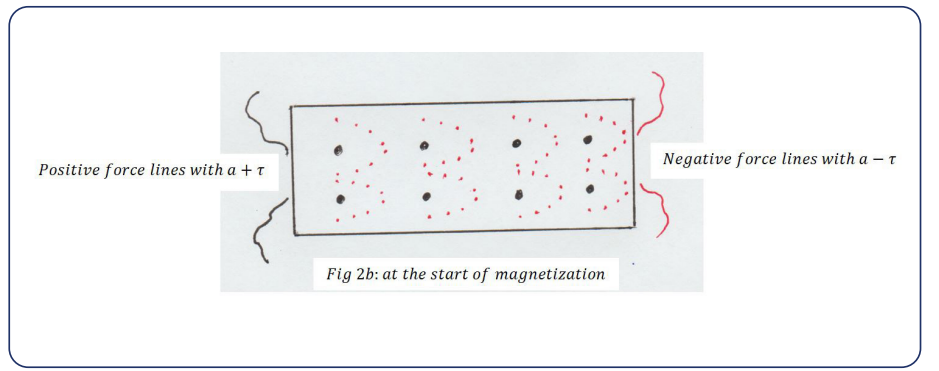

In Figure $2 b$, at the start of magnetization at $t=0$ seconds, the negative forces are coming out from the exposed inner electron (-e) based negative or north magnetic pole $\mathrm{N}$ and these negative forces are experiencing a negative torque $(-\tau)$ passed on from the negative torque ($\tau)$ of the exposed inner electron based negative or north magnetic pole N.
The exposed proton $(+e)$ based positive or south magnetic pole $S$ has the positive forces coming out with a positive torque $(+\tau)$ passed on from the positive torque $(+\tau)$ of the exposed protons of the south magnetic pole $\mathrm{S}$.

These negative and positive forces also exist at the inter-atomic intra-magnetic level when magnetized and even inside the magnet there is a much greater coulombs force as the coulombs force law is applicable.

These negative forces with a negative torque $(-\tau)$ from $\mathrm{N}$ travel towards the positive or south magnetic pole $S$ and the positive forces with a positive $(+\tau)$ torque from $S$ travel towards the negative magnetic pole $\mathrm{N}$ and owing to their opposing torque they entwine to manifest as a single magnetic force erroneously called the single fundamental force of magnetism.

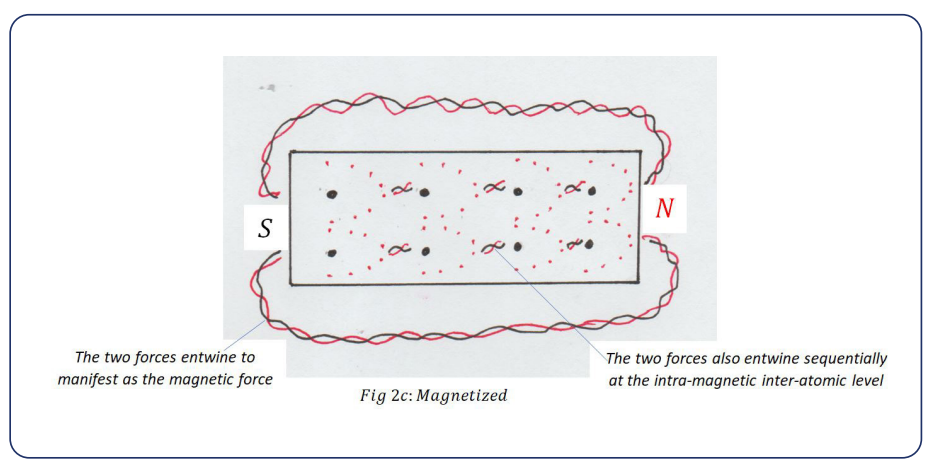

In above figure $2 \mathrm{c}$ at $\mathrm{t}>0$ seconds, the negative forces are coming out of the negative or north magnetic pole $\mathrm{N}$ with a negative torque $(-\tau)$ and are headed for the exposed positive or south magnetic pole $\mathrm{S}$ and the positive forces are coming out of the exposed south magnetic pole $S$ with a positive torque $(+\tau)$ and are headed for the negative or north magnetic pole $\mathrm{N}$.

The intra-magnetic force will get stronger with cooling owing to decreased distance and no magnetic flux whatsoever can be expelled laterally. Cooling will facilitate increased magnetization as the electrons and protons of neighboring atoms get closer to each other and this will manifest from the exposed magnetic ends only.

The magnetic force of attraction is a combination of the negative force from the north magnetic pole $\mathrm{N}$ with a negative torque $-\tau$ and the positive force from the south magnetic pole $\mathrm{S}$ with a positive torque $+\tau$.

Owing to the opposing torque between the forces from the north magnetic pole $\mathrm{N}$ and from the south magnetic pole $\mathrm{S}$, these two forces of attraction entwine with each other as in Figure $2 \mathrm{c}$ and appear to be a single magnetic force of attraction both:

1-at the inter-magnetic intra- magnetic level, this attractional force gets stronger with cooling and no lateral expulsion of magnetic flux possible although greater magnetization possible due to decreased distance $^{5}$. and

2-at the extra-magnetic level it does manifest from the two ends of a magnet. 


\section{Amperes Law and Amperes right hand rule}

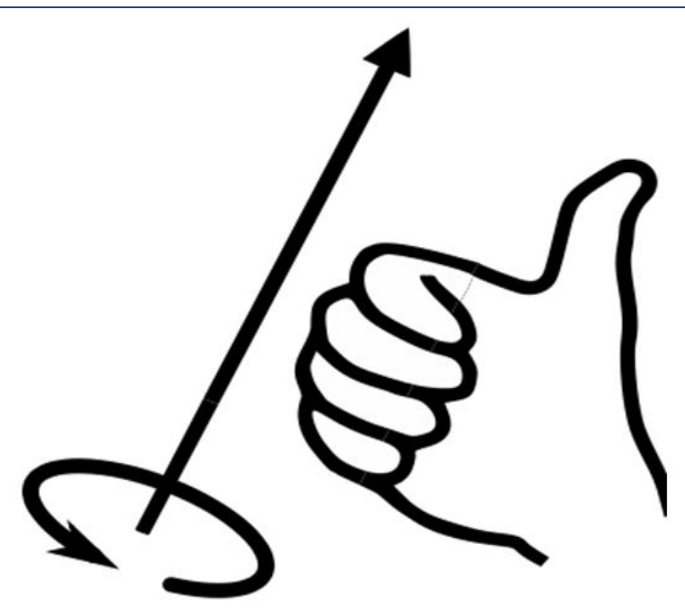

Figure2d

According to Amperes right hand grip rule 1826 as in Figure 2d, if the fingers of the right hand are curled around the coil in the direction of current flow (conventional current is flow of positive charge) and the thumb points in the overall direction of the positive current flow, then the thumb points in the direction of the north magnetic pole N.

According to Gill's electronic theory of magnetism 1964 and as discussed above, the direction of the thumb points to extra inner electrons of the iron core atom subjected to a pull in the direction of the thumb with a negative torque $(-\tau)$ in the direction of the curled fingers owing to the centripetal force of the direct electric current in the conducting coil.

Thus, the north magnetic pole is the same as the exposed inner electron dependant negative magnetic pole $\mathrm{N}$.

Corollary (1) Gill's modified right hand grip rule for a magnet: If the fingers of the right hand are curled around a magnet and the thumb is pointed in the direction of the north/negative magnetic pole $\mathrm{N}$ of the magnet, then the non-moving inner electrons at the north magnetic pole will have a negative torque $(-\tau)$ in the direction of the curl of the fingers.

Applying Ampere's 1826 right hand grip rule combined with Gill's electronic theory of magnetism 1964 explains the above result of magnetization in an electromagnet.

A permanent magnet would have the same situation of two forces headed for each other from the two ends of the magnet manifesting as a single magnetic force which we erroneously call the single fundamental force magnetism.

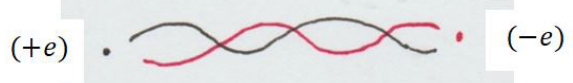

Fig $2 e$ : entwined forces between a proton and an electron
In Fig 2e, the force from the negative source (-e) with negative torque $(-\tau)$ heads for the positive source and the force from the positive source $(+e)$ with a positive torque $(+\tau)$ heads for the negative source and this entwined combination of the electron (-e) based negative force and the proton based $(+e)$ positive force is later the travelling force.

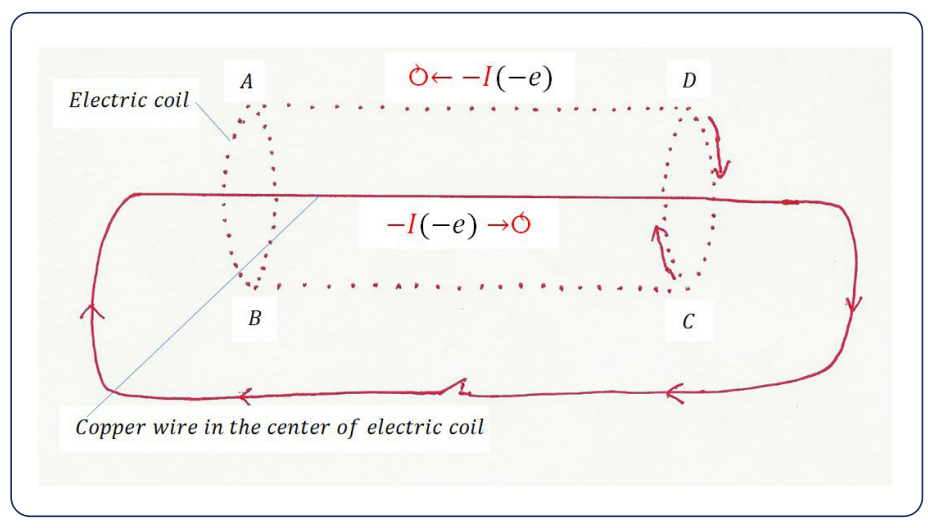

Figure $2 \mathrm{f}$

Figure $2 \mathrm{f}$ is the same as Figure $2 \mathrm{a}$ except that instead of an iron piece which gets magnetized in Figure 2a, we have placed a copper wire in the middle of the electric coil.

The electrons (-e) flowing in the electric coil are repelling and rotating the outer free valence electrons (-e) in the central copper wire as a direct electric current $-\mathrm{I}(-\mathrm{e}) \rightarrow \mathrm{O}$ in a direction opposite to the original electron flow in the electric coil.

\section{Converting between Newtons and Teslas}

Gill'selectronic theory of magnetism 1964 simplifies the conversion between the Coulomb force law in Newtons and the magnetic force called the Tesla.

If we have an attraction force of one newton between the two magnetic poles when they are one meter apart, then we call it a magnetic force of one Tesla.

Applying Gill's electronic theory of magnetism 1964, the force of attraction between the two poles of a magnet, $\mathrm{n}(-\mathrm{e})$ coulombs at the negative magnetic pole $\mathrm{N}$ and $\mathrm{n}(+\mathrm{e})$ coulombs at the positive magnetic pole S can be calculated by applying Coulomb's law 1784 .

$\mathbf{F}=\frac{\mathbf{k}(\mathbf{n}(-\mathbf{e}))(\mathbf{n}(+\mathbf{e}))}{\mathbf{d}^{2}}$ newtons $=-\frac{\mathbf{k n}^{2} \mathbf{e}^{2}}{\mathbf{d}^{2}}$ newtons

The negative or North magnetic pole $\mathrm{N}$ has an exposed negative charge $n(-e)$ coulombs where $n$ is the number of exposed inner electrons at magnetization.

The positive or South magnetic pole $\mathrm{S}$ has an exposed positive magnetic charge $n(+e)$ coulombs where $n$ is the number of exposed protons at magnetization.

Rearranging, the above equation becomes

$$
\mathbf{n}^{2}=-\frac{\mathrm{Fd}^{2}}{\mathbf{k e}^{2}} \text { or in units it is } \frac{\mathrm{Nm}^{2}}{\left(\mathrm{Nm}^{2} \mathbf{C}^{-2}\right) \mathbf{C}^{2}}
$$


If $\mathrm{F}=-1$ newton and $\mathrm{d}=1$ meter, the magnetic force is one Tesla between the two magnetic poles. Substituting these values, the above equation becomes

$$
\begin{aligned}
& \mathbf{n}^{2}=\frac{1}{\mathbf{k e}^{2}} \text { or } \\
& \mathbf{n}=\frac{1}{\sqrt{\mathbf{k}} \cdot \mathbf{e}}=(\sqrt{\mathbf{k}} \cdot \mathbf{e})^{-1} .
\end{aligned}
$$

Coulomb's constant $\mathrm{k}=8.99 \times 10^{9} \mathrm{Nm}^{2} \mathrm{C}^{-2}$ where $\mathrm{N}$ is for newtons, $\mathrm{m}$ is in meters and $\mathrm{C}$ is for coulombs and $1 \mathrm{e} \approx 1.6 \times 10^{-19} \mathrm{C}$.

Applying these, we get

$$
\mathrm{n}=\frac{1}{\sqrt{\mathrm{k} . \mathrm{e}}}=\frac{1}{\sqrt{\left(8.99 \times 10^{9}\right)} .\left(1.6 \times 10^{-19}\right)}=6.591741555135 \times 10^{13} \approx 6.6 \times 10^{13}
$$

Tesla: If we have a negative or north magnetic pole $\mathrm{N}$ with $6.6 \times 10^{13}$ exposed inner electrons and at one meter, we have a positive or south magnetic pole $\mathrm{S}$ with $6.6 \times 10^{13}$ exposed protons, then the attractional magnetic force between the two magnetic poles is one newton or one Tesla. In addition, the negative electrons (-e) at the north magnetic pole $\mathrm{N}$ have a negative torque $(-\tau)$ and the positive protons $(+)$ at the south magnetic pole $S$ have a positive torque $(+\tau)$.

\section{Discussion of Gauss' Law for a positive and a negative source ${ }^{6}$}

Gauss' Law and the +e and -e forces

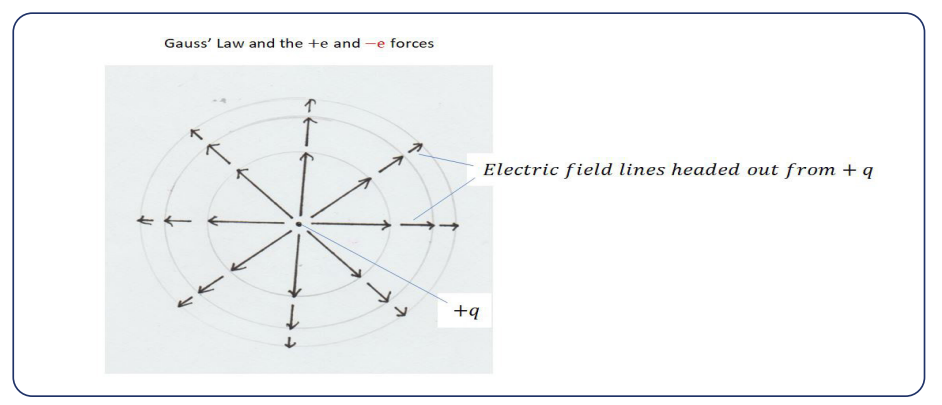

Figure 3a

Gauss' law states that the electric field $\mathrm{E}$ heads out in all directions from the positive charge $+\mathrm{q}$ placed at the center and is called the positive flux of the electric field.

Gauss' Law: $\varepsilon_{0} \oint \mathrm{E} \cdot \mathrm{dA} \cos \theta=+\mathrm{q}$.

As $\cos \theta=\cos 0^{\circ}=1$ and $\varepsilon_{0}$ is the permittivity constant we get.

$\varepsilon_{\oint} \oint \mathrm{E} . \mathrm{dA}=+\mathrm{q}$, as electric field $\mathrm{E}$ is a constant for this situation, we could write it as

$\varepsilon_{0} E \oint d A=+q$, and as $\oint d A=4 \pi r^{2}$ for a sphere, we get

$\varepsilon_{0} E 4 \pi r^{\wedge} 2=+q$. Rearranging this we get the electric field

$$
\mathrm{E}=\frac{1}{4 \pi \varepsilon_{0}} \cdot \frac{+\mathrm{q}}{\mathrm{r}^{2}}=+\frac{1}{4 \pi \varepsilon_{0}} \cdot \frac{\mathrm{q}}{\mathrm{r}^{2}}
$$

Figure 3a has positive field lines emanating from a positive point charge $(+q)$ at the center and the flux is positive. Another positive charge placed in this field will be repelled along the positive electric field lines.

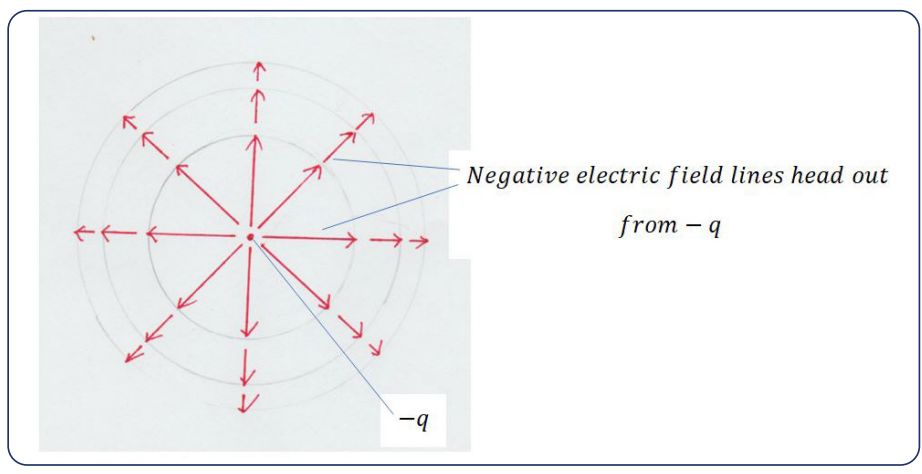

Figure $3 b$

Modified Gauss' law shows that a negative electric field $-E$ heads out in all directions because of a negative charge $-\mathrm{q}$ at the center as in Figure $3 b$.

Modified Gauss' Law: $\varepsilon_{\circ} \oint(-\mathrm{E}) \cdot \mathrm{d} A \cos \theta=-\mathrm{q}$.

As $\cos \theta=\cos 0^{\circ}=1$ and $\varepsilon_{\mathrm{o}}$ is the permitivity constant we get

$\varepsilon_{0} \oint(-E) . d A=-q$, as the negative electric field $-E$ is a constant, we could write it as

$\varepsilon_{0}(-E) \oint d A=-q$, and as $\oint d A=4 \pi r^{2}$ for a sphere, we get

$\varepsilon_{0}(-E) 4 \pi r^{2}=-q$. Rearranging this we get the electric field

$-\mathrm{E}=\frac{1}{4 \pi \varepsilon_{0}} \cdot \frac{-\mathrm{q}}{\mathrm{r}^{2}}=-\frac{1}{4 \pi \varepsilon_{0}} \cdot \frac{\mathrm{q}}{\mathrm{r}^{2}}$ Equation 3b

Figure $3 \mathrm{~b}$ has negative electric field lines emanating from a negative point charge $(-q)$ at the center as a negative flux and another negative charge placed in this negative electric field will be repelled along the negative field lines.

Positive charge forces spread out along positive electric field lines. Negative charge forces spread out along negative electric field lines. Whenever there is an attraction between the positive and the negative electrical sources, there are two types of force lines headed for the opposing sources as in Figure 3c:

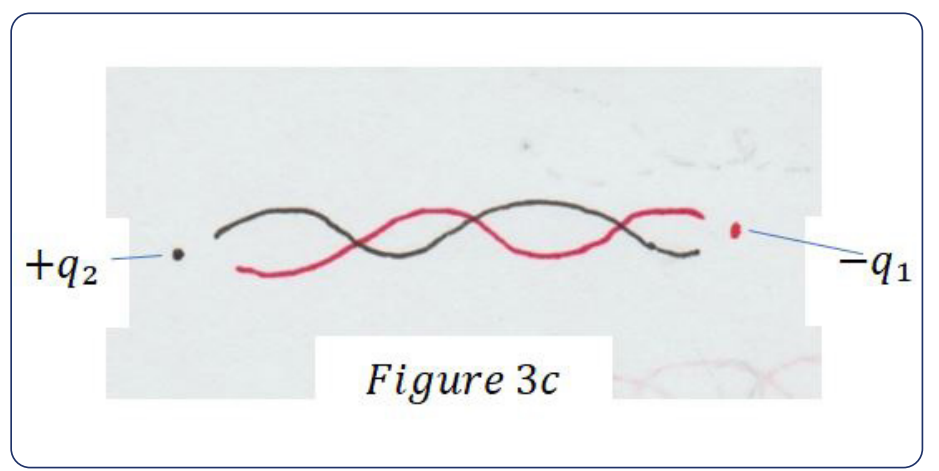

The positive source $+\mathrm{q}_{2}$ with a positive torque $(+\tau)$ has the positive force lines with a positive $(+\tau)$ head for the negative source $-q_{1}$.

The negative source $-q_{1}$ with a negative torque $(-\tau)$ has the negative force lines with a negative $(-\tau)$ head for the positive source $+\mathrm{q}_{2}$.

Although the merged result makes it appear as a single electrical force, the existence of Positive and Negative electric fields maybe noted. 
The combined entwined attractional force between $\left(-\mathrm{q}_{1}\right)$ and $\left(+\mathrm{q}_{2}\right)$ along electric field lines results in:

Coulombs force law $\mathrm{F}=-\frac{1}{4 \pi \varepsilon_{0}} \cdot \frac{\left(-\mathrm{q}_{1}\right)\left(+\mathrm{q}_{2}\right)}{\mathrm{r}^{2}} \ldots \ldots \ldots \ldots$. Equation $3 \mathrm{c}$

While discussing the Capacitor physics, the author is pointing out that when a positive electric field line from a positive source encounters a negative field line from a negative source, both those field lines head for the opposite source and entwine with each other.

\section{$(\text { The Coil Effect })^{\mathrm{n}}$}

Electrical force on a proton in the central copper wire from the circular conducting coil.

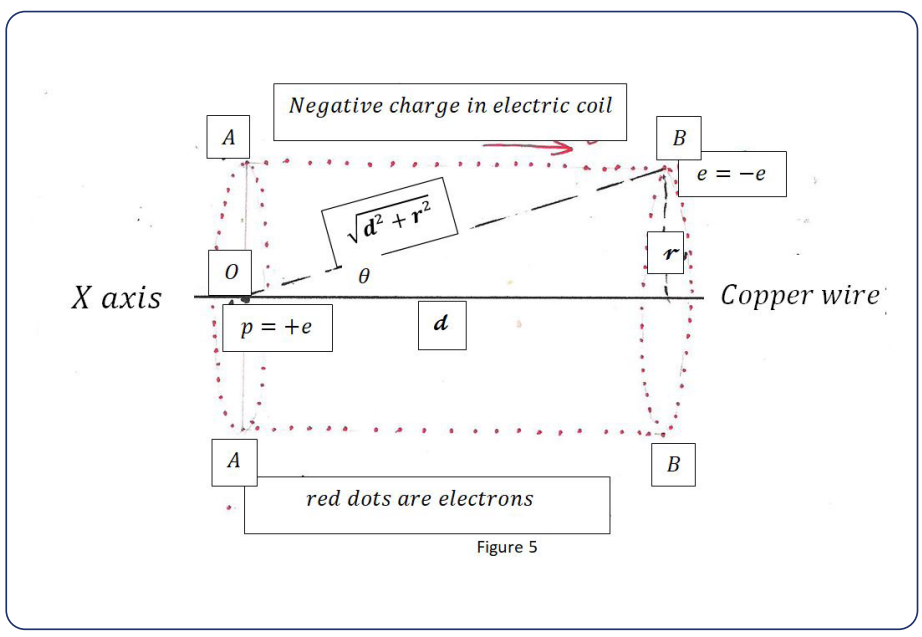

Figure 5

$\mathrm{AA}$ to $\mathrm{BB}$ is a cylindrical conducting electric coil conducting direct negative electric current from point A to point B. A copper wire has been placed in the center of the coil along the $\mathrm{X}$-axis. At the point $\mathrm{O}$ of the central copper wire along the $\mathrm{X}$ axis, we have placed an exposed proton of an atom of the copper wire with a charge $\mathrm{p}=+\mathrm{e}$. As the mild solar electrical current is flowing through the coil, we have electrons flowing from $\mathrm{A} \rightarrow \mathrm{B}$ in the copper coil and a free valence electron with a charge -e is placed at point $\mathrm{B}$ in the conducting copper coil.

The distance $\mathrm{A} \rightarrow \mathrm{B}=\mathrm{d}$ meters and $\mathrm{r}$ meters is the radius of the

coil. Applying Pythagoras theorem, the distance $\mathrm{OB}=\sqrt{ }\left(\mathrm{d}^{\wedge} 2+\mathrm{r}^{\wedge} 2\right)$.

The force of attraction between proton $\mathrm{p}=+\mathrm{e}$ at point $\mathrm{O}$ and electron $\mathrm{e}=-\mathrm{e}$ at point $\mathrm{B}$ is

$$
\mathbf{F}=\frac{\mathbf{k}(+\mathbf{e})(-\mathbf{e})}{\left(\sqrt{\left.\mathbf{d}^{2}+\mathbf{r}^{2}\right)^{2}}\right.}=-\frac{\mathbf{k e}^{2}}{\mathbf{d}^{2}+\mathbf{r}^{2}} \text { newtons(Coulombs law) }
$$

Where $\mathrm{k}$ is the Coulomb's constant. The bold letters are vectors.

The minus sign indicates attraction between the flowing negative electron at point $\mathrm{B}$ and the exposed positive proton $(+\mathrm{e})$ of an atom of the central copper wire at point $\mathrm{O}$.

$\theta$ is the angle between $\mathrm{OB}$ and the $\mathrm{X}$ axis and the dot product vector of $\mathrm{OB}$ along the $\mathrm{X}$ axis is

$$
\mathbf{F} \cos \theta=-\frac{\mathbf{k e}^{2}}{\mathbf{d}^{2}+\mathbf{r}^{2}} \cos \theta=-\frac{\mathbf{k e}^{2}}{\left(\mathbf{d}^{2}+\mathbf{r}^{2}\right)} \cdot \frac{\mathbf{d}}{\sqrt{\mathbf{d}^{2}+\mathbf{r}^{2}}}
$$

As $d$ is exceptionally large compared to $r$, then $r^{2}$ is still smaller and can be ignored and the above vector along $\mathrm{X}$-axis is

$$
\mathbf{F} \cos \theta=-\frac{\mathbf{k e}^{2}}{\mathbf{d}^{2}} \cdot \frac{\mathbf{d}}{\sqrt{\mathbf{d}^{2}}}=-\frac{\mathbf{k e}^{2}}{\mathbf{d}^{2}} .
$$

As the electrons move from $\mathrm{A}$ to $\mathrm{B}, \mathrm{d}$ varies from $\mathrm{d}=0$ at $\mathrm{A}$ to $d=d$ meters at $B$ and if $d_{x}$ is an infinitesimally small distance along $\mathrm{A} \rightarrow \mathrm{B}$, then the vector along the $\mathrm{X}$ axis becomes

$$
F=\int_{0}^{d}-\frac{k^{2} d_{x}}{d^{2}}=-k^{2} \int_{0}^{d} \frac{d_{x}}{d}
$$

The derivative of $\log d=1 / d$, so the anti-derivative of $1 / d=\log d$ and integrating from 0 to $\mathrm{d}$ we get

$$
F=-\mathbf{k e}^{2}[\log d]_{0}^{d}=-\mathrm{ke}^{2}(\log \mathrm{d}-\log 0)=-\mathrm{ke}^{2} \cdot \log \mathrm{d}
$$

newtons............Equation 5 a

The number of electrons rotating in a circular manner in every coil is a at any particular time. In case of copper wire, each atom has one free valence electron, so a also represents the number of copper atoms in the circular coil, and a depends on radius $r$ and the total a will depend on the number of coils at each level and the total vector from Equation 5a along positive $\mathrm{X}$-axis direction from $\mathrm{A} \rightarrow \mathrm{B}$ is

Electrical force $\mathbf{F}($ total $)=-\mathbf{k a e}^{2} \log$ newtons---Equation $5 b$

This increased central electrical force will manifest as a positive electric current along the central copper wire or better still as a negative electron based electric current flowing in the opposite direction.

In actuality, the loose valence electrons in the central copper wire flow in the direction opposite to the direction of the negative electric current in the coil from $\mathrm{B} \rightarrow \mathrm{A}$.

This negative electric current in the central wire will by repulsion from the electrons in the coil will also be rotating in a direction opposite to the direction of negative electric current in the coil by repulsion.

Thus, the negative electric current in the central copper wire flows and rotates from $\mathrm{B} \rightarrow \mathrm{A}$ as a multiple of the direct electric current in the coil.

The increased positive force on the central copper wire is due to the 'coil effect' of the moving free valence electrons in the coil as an electric current and this has become a significant multiple at each level and increasing the distance $\mathbf{d}$ of the coil as far as technically possible would also help.

As the atoms in the central metallic wire are in a homogeneous medium, the calculations can be done from the central axis of the central wire for all the atoms in the central metallic wire. 
The entwined attractional force is between the electron $(\mathrm{e}=-\mathrm{e})$ at point $\mathrm{B}$ and the proton in copper wire $(\mathrm{p}=+\mathrm{e})$ at point $\mathrm{O}$. Coulombs force law does the rest.

Conclusion: Even a mild imperceptible electric current in a coil maybe multiplied by the coil effect to produce useful renewable energy in the central wire.

Applications: Electrical Engineering could go a long way in helping capture this mild electrical energy which is everywhere?

\section{The Cyclotron and The Electric Motor}

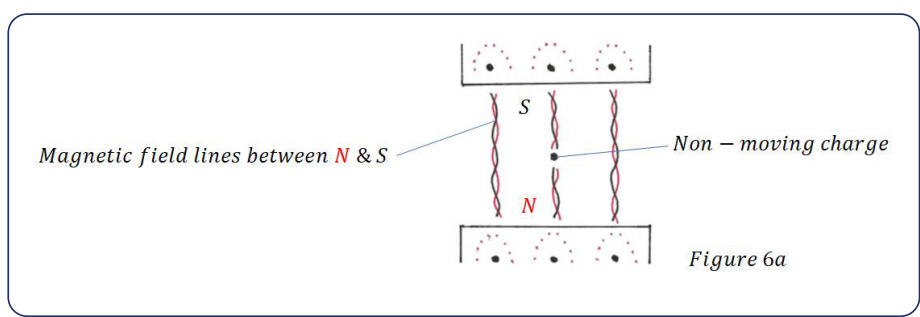

A non-moving charge in a magnetic field $\mathbf{B}$ will not move.

However, a moving positive ion will be repelled perpendicular $\perp$ to $\mathbf{B}$ by the positive torque $(+\tau)$ originating from the positive exposed proton $(+e)$ based south magnetic pole $\mathrm{S}$ ultimately resulting in the positive charge moving in a clockwise direction.

A moving negative ion is repelled by a negative torque $(-\tau)$ originating from the exposed electron (-e) based north magnetic pole $\mathrm{N}$ perpendicular $\perp$ to $\mathbf{B}$ in the opposite anti-clockwise direction.

This property of magnetic force $\mathbf{B}$ combination will be applied to explain the working of the Cyclotron and the Electric motor.

\section{The Cyclotron}

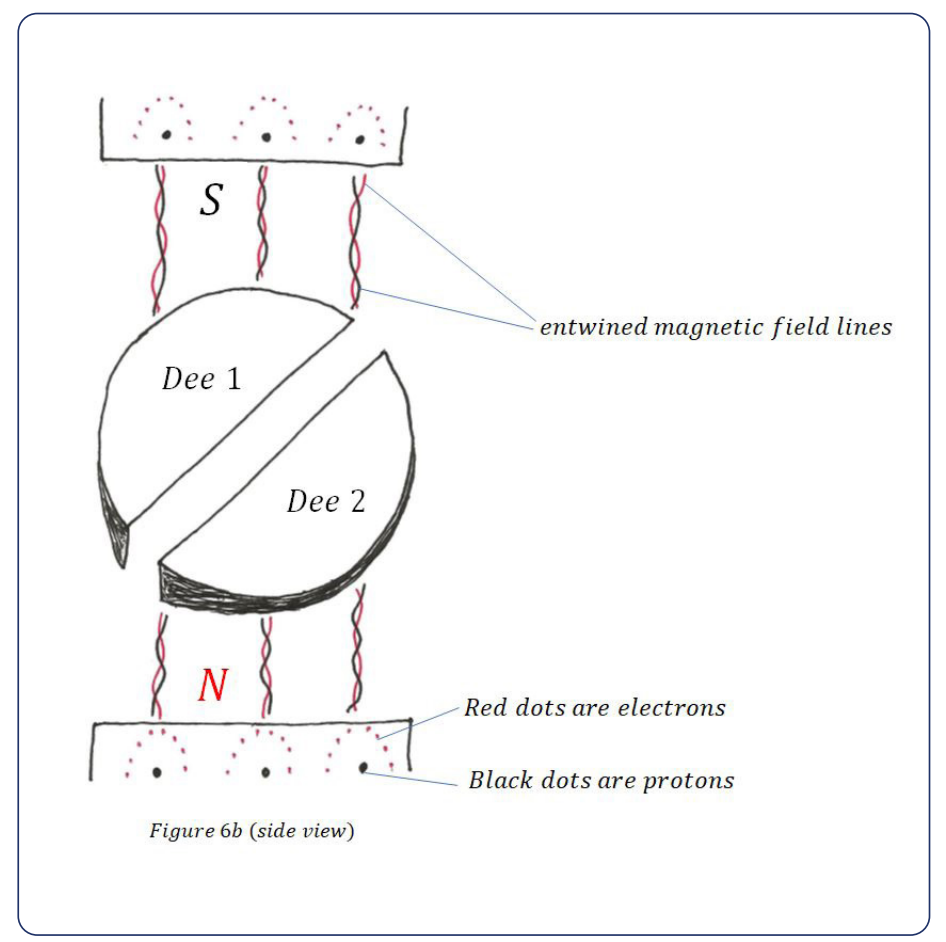

In Figure 6b, the two semi-circular plates Dee1 and Dee2 with a gap between them are placed between the two magnetic poles of an electro-magnet to which 'Gill's electronic theory of magnetism 1964' has been applied.

The positive torque $(+\tau)$ component of the magnetic force $\mathbf{B}$ from positive south magnetic pole $\mathrm{S}$ causes a repulsive effect on a moving positively charged ion/proton $(+e)$. The negative torque $(-\tau)$ component of the magnetic force $\mathbf{B}$ causes a repulsive effect on a moving negatively charged electron (-e), even when these charges are flowing freely as an electric current on the surface of an electric wire.

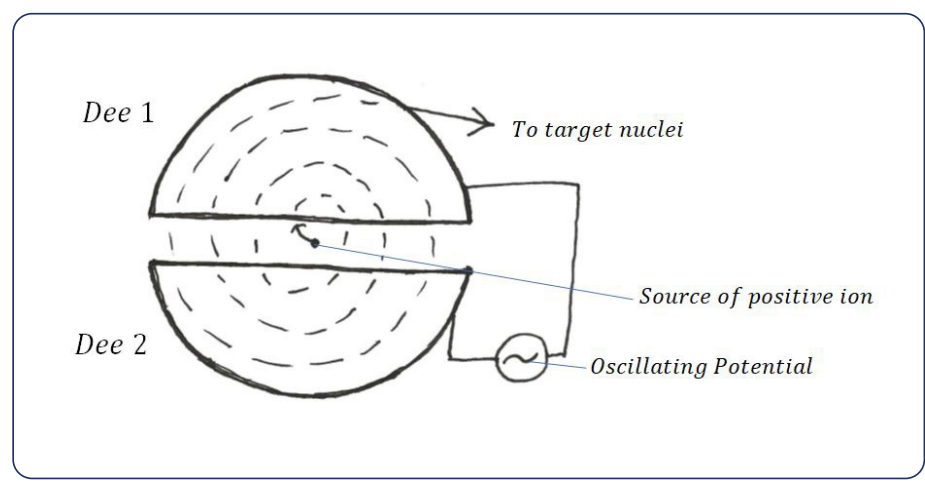

Figure 6c

Gill's electronic theory of magnetism 1964 has been applied to the electromagnet of the cyclotron.

The magnetic field $\mathbf{B}$ has two components and the positive torque $(+\tau)$ from the positive or south magnetic pole $\mathrm{S}$ will repel the moving positive ion with a mass $\mathrm{m}$ and charge $+\mathrm{q}$ with a velocity $\mathrm{v}_{1}$ perpendicular $(\perp)$ to both the velocity $\mathrm{v}_{1}$ and also perpendicular $(\perp)$ to the magnetic field $\mathbf{B}$ applying Ampere's right-hand rule.

The force $\mathrm{F}=\mathrm{qv}_{1} \mathrm{~B} \sin 90^{\circ}=\mathrm{qv}_{1} \mathrm{~B}$ which as a centripetal force will give us a radius $R_{1}$.

The cross-product Lorentz Force Law $F=q v_{1} \times B$ is not needed.

$q v_{1} B=\frac{m v_{1}^{2}}{R_{1}}$ or $R_{1}=\frac{m v_{1}}{q B}$ and $v_{1}=\frac{q B R_{1}}{m}$.

Time period $T=\frac{2 \pi R_{1}}{v_{1}}=\frac{2 \pi R_{1}}{q B R_{1}} m=\frac{2 \pi m}{q B}$.

Frequency $f=1 / T=q B / 2 \pi m$ which is synchronized with the oscillating potential as shown in Figure $6 \mathrm{c}$ and the resonance frequency must match. The oscillating potential keeps changing the polarity of the two plates Dee1 and Dee2 and the velocity of the charged particle keeps on increasing every time it reaches between Dee1 and Dee2.

$$
\begin{aligned}
& q V=K E_{2}-K E_{1}=\frac{1}{2} m\left(v_{2}^{2}-v_{1}^{2}\right) \quad \text { or } \quad \frac{2 q V}{m}=v_{2}^{2}-v_{1}^{2} \\
& v_{2}=\sqrt{v_{1}^{2}+\frac{2 q V}{m}}
\end{aligned}
$$

The radius of the charged particle in Deel is $R_{1}=\left(m v_{1}\right) / q B$ and in Dee 2 the $\mathrm{v}_{2}$ of the charged particle has increased radius $\mathrm{R}_{2}=\left(\mathrm{mv}_{2}\right) / \mathrm{qB}$. 
Repeating this again and again results in the charged particle develop a high velocity which could be used for experiments and production of useful compounds.

Conclusion: it has been shown with the help of Gill's electronic theory of magnetism 1964, how the torque component of $\mathbf{B}$ is responsible for the circular motion of a moving positive or negative ion in a magnetic field in a cyclotron and ultimately results in the particle having a high velocity.

\section{The Electric Motor}

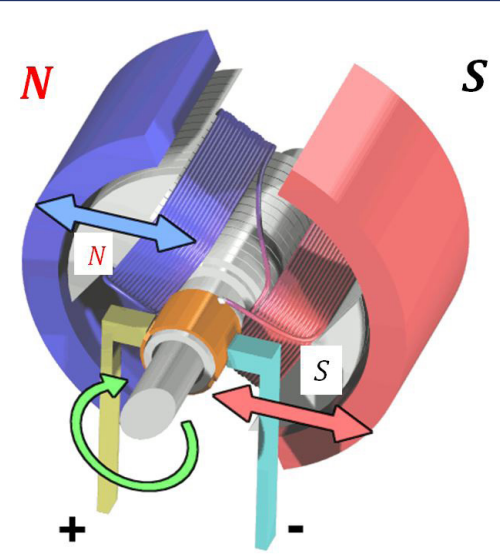

Figure7a Electric Motor (Photograph from Wikipedia)

Figure 7a shows an electric motor which is an electrical machine that converts electrical energy into mechanical energy and reversing this results in an electrical generator.

The electric coil around the Rotor is placed between the negative electron (-e) dependant north magnetic pole $\mathrm{N}$ and the positive proton $(+e)$ dependant south magnetic pole $\mathrm{S}$ of the permanent magnet or Stator. The Commutator flips the direct electric current every $180^{\circ}$.

A moving negative electron (-e) in the direct electric current is repelled by a negative torque $(-\tau)$ originating from the exposed electron (-e) based north magnetic pole $\mathrm{N}$ perpendicular $\perp$ to $\mathbf{B}$ in the opposite anti-clockwise direction.

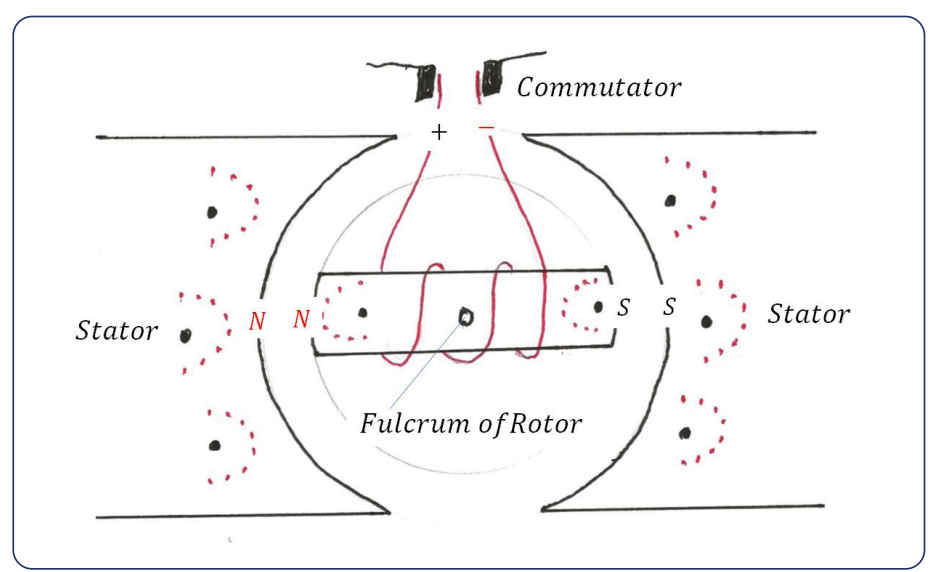

Figure $7 \mathrm{~b}$ (electric motor) top view with Gill's electronic theory of magnetism 1964

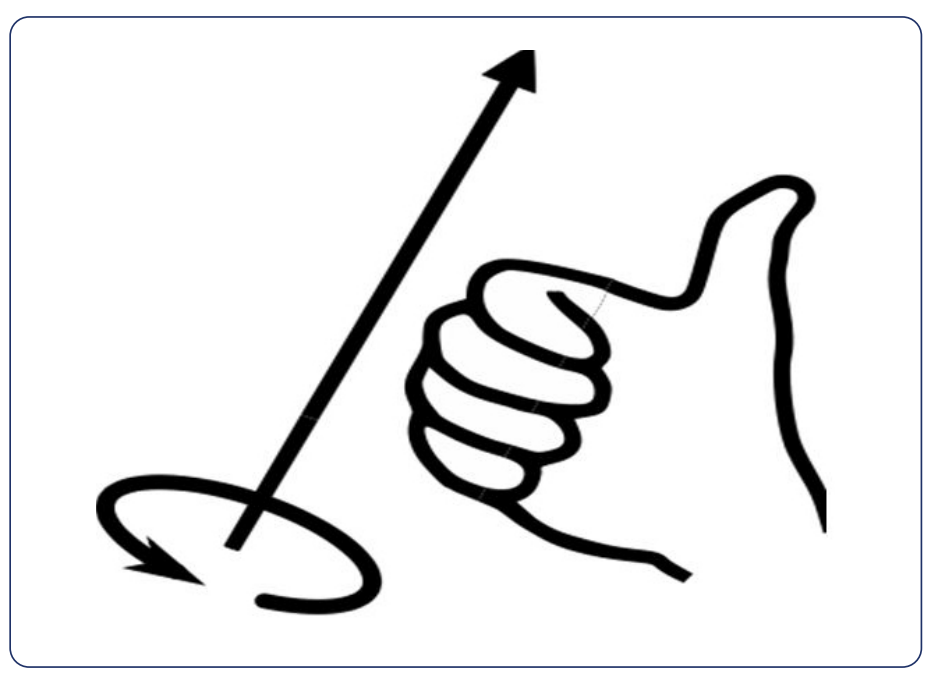

Figure 7c

Combining Gill's electronic theory of magnetism 1964 with Ampere's right-hand rule 1826, the north magnetic pole is the same as the exposed inner electron dependant negative or north magnetic pole $\mathrm{N}$ with a negative torque $(-\tau)$.

A moving negative electron (-e) is repelled by the negative torque $(-\tau)$ originating from the exposed electron (-e) based north magnetic pole $\mathrm{N}$ perpendicular $\perp$ to $\mathbf{B}$ in the opposite anti-clockwise direction.

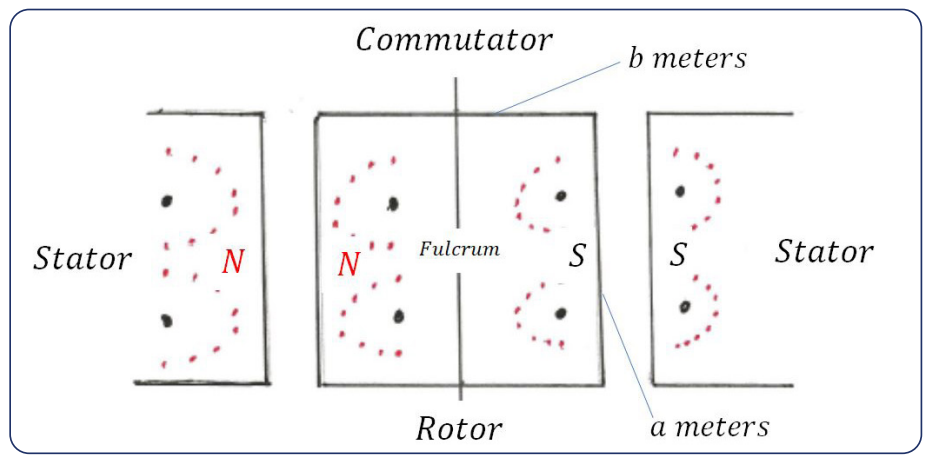

Figure 7d (electric motor) frontal view with rectangular coil

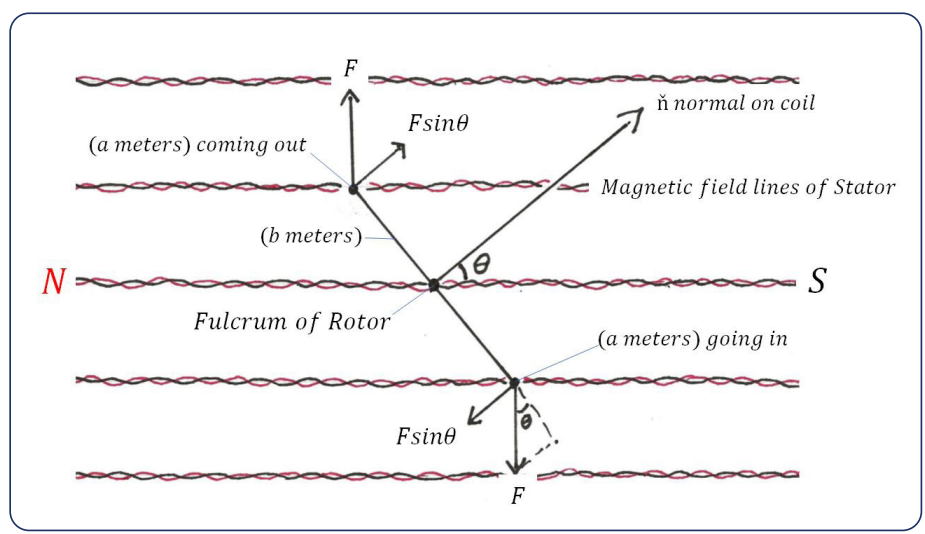

Figure 7 e $X$-Y plain

Figure $7 \mathrm{~d}$ is a frontal view of a single rectangular electric coil around the Rotor with two horizontal components of (b meters) with a fulcrum in its middle. On either side, there is a vertical component of (a 
meters) perpendicular to the magnetic field lines of the Stator.

The magnetic force B from the Stator is always perpendicular $(\perp)$ to the moving electrons as a direct electric current flowing in the two (a meters) components of the coil.

The negative torque $(-\tau)$ torque force from B repels the moving electrons perpendicular $\perp$ to $\mathrm{B}$ which causes a lateral force $\mathrm{F}$ around the fulcrum from both (a meter) components of the coil.

The (b meter) components being opposite to each other neutralize each other and have no effect on rotation of the Rotor.

Gill's electronic theory of magnetism 1964 explains that the negative or electron based north magnetic pole $\mathrm{N}$ has a negative torque $(-\tau)$ passed on to the magnetic field lines of the Stator. This manifests on moving electrons in the electric coil in the (a meter) arms and results in a force $\mathrm{F}$ perpendicular $\perp$ to the magnetic field lines of $\mathrm{B}$ and perpendicular $\perp$ to the direct electric current flow in the coil and Ampere's right-hand rule supports this explanation.

Calculating Torque $(\tau)$ on a direct rectangular electric current loop:

In Figure 7e, an electric motor represented by a single rectangular electric coil is placed between the two permanent magnetic poles $\mathrm{N}$ and $\mathrm{S}$ of the Stator with a central rod/fulcrum at an equal distance from the two permanent magnetic poles of the Stator.

In Figure 7e, around the Rotor, there is one (a meters) component of the electric wire with direct electric current going into the board at one end of (b meters) and at its other end the second (a meters) component of the electric wire with direct electric current is coming out of the board.

Both lateral components are always perpendicular $\left(\perp=90^{\circ}\right)$ to the magnetic field lines of the permanent magnet or Stator.

The flowing electrons as a direct electric current are repelled by the perpendicular $\perp$ negative torque (- $\tau$ ) of the permanent magnet of the Stator along the (a meters) with a force $F$.

In Figure 7e, the right hand rule shows force on the (a meters) component of the wire with electrons spinning and moving out of the page in a Stator magnetic field is $\mathrm{F}$ upwards and a downward force $\mathrm{F}$ at the other end of $\mathrm{b}$ meters as (a meters) is now going into the page because of a $180^{\circ}$ change in direction of the electric coil and the pivot point in the center of $b$ meters causes the coil to rotate for $180^{\circ}$.

Force F because of the Stator:

As (a meters) of the Rotor coil on opposing sides is always perpendicular $\left(\perp=90^{\circ}\right)$ to the magnetic field of the Stator.

$\mathrm{F}=\mathrm{iaB} \sin 90^{\circ}=\mathrm{iaB}$

i amperes is the direct electron based electric current in the coil.

$\mathrm{B}=\mathrm{k} \cdot \frac{\mathrm{n}(-\mathrm{e}) \mathrm{n}(+\mathrm{e})}{\mathrm{b}^{2}}=-\mathrm{k} \cdot \frac{(\mathrm{ne})^{2}}{\mathrm{~b}^{2}}$ teslas is the permanent magnetic force of the Stator. $\mathrm{b}$ meters $\approx$ distance between the two poles of the Stator.

$\mathrm{n}(-\mathrm{e})$ is the number of exposed electrons $\mathrm{N}$ of the Stator.

$\mathrm{n}(+\mathrm{e})$ is the number of exposed protons $\mathrm{S}$ of the Stator.

Normal vector $\check{n}$ of the coil is always perpendicular $\perp$ to the coil at 90 degrees.

$\theta$ is the angle between the normal vector $\check{n}$ and the magnetic field of the Stator.

Owing to the fulcrum in the middle of ( $b$ meters), the moment arm is $\mathrm{b} / 2$ meters. Torque $\tau=\mathrm{b} / 2 \mathrm{~F} \sin \theta$.

As $\mathrm{F}=\mathrm{iaB}$, substituting this in the above equation, we get

Torque $\tau=\mathrm{b} / 2 \mathrm{~F} \sin \theta=\mathrm{b} / 2 \mathrm{iaB} \sin \theta$ is for one of the two (a meters) arm.

Add to this the torque on the opposite (a meters) arm we get:

Total torque on a current carrying loop

$\tau=\mathrm{b} / 2 \mathrm{iaB} \sin \theta+\mathrm{b} / 2 \mathrm{iaB} \sin \theta=\mathrm{abiB} \sin \theta$

If $\mathrm{N}$ is the number of coils and $\mathrm{ab}=\mathrm{A}$ the surface area of the coil then:

Total torque $\tau=\mathrm{NabiB} \sin \theta=(\mathrm{NiA}) \mathrm{B} \sin \theta$ and the unit is newton.meters.

i amperes is the direct electron based electric current in the coil.

A is the surface area of the coil (applies for any type of surface).

B teslas is the magnetic force between the two poles of the permanent magnet.

As $\sin \theta$ varies from $0^{\circ}$ to $180^{\circ}$ and at $\sin 90^{\circ}=1$ gives the maximal torque.

The Rotor will start rotating a little above $\sin 0^{\circ}=0$ and continues until it reaches $\sin 180^{\circ}=0$ when the rotor will try to stop but at this stage the Commutator will reverse the direct electric current around the Rotor and it will continue rotating.

In Figure 7d, the upper and lower (b meters) components of the Stator have no direct effect on the rotation of the Rotor.

The electromagnet of the Rotor in the center always tries to align with the Stator which is a permanent magnet. It tries to make its negative or north magnetic pole $\mathrm{N}$ to be aligned with the Stators positive or south magnetic pole $\mathrm{S}$ by attraction and vice versa.

The forces between the (a meters) vertical components and the Stator will force rotation by repulsion whenever the magnetic poles of the rotor $\mathrm{N}$ are opposite to the permanent magnet pole $\mathrm{N}$ of the Stator. 
The Commutator: After a rotation of $180^{\circ}$, the electric motor would stop when the magnetic poles of the Rotor $\mathrm{N}$ are opposite to the permanent magnet pole $\mathrm{S}$ of the Stator. At this stage, the Commutator flips the direct electric current in the electric coil of the Rotor to change the polarity of the electromagnet in the Rotor and the rotation of the electric motor is repeated.

Conclusion: The rotation of an electric motor with the dot product torque force $\mathrm{F}$ from the magnetic field lines originating from the the Stator has been explained with the help of Gill's electronic theory of magnetism 1964 and Ampere's right-hand rule 1826. Cross product of Henrik Lorentz 1893 not needed.

\section{The Capacitor (Charge reservoir)}

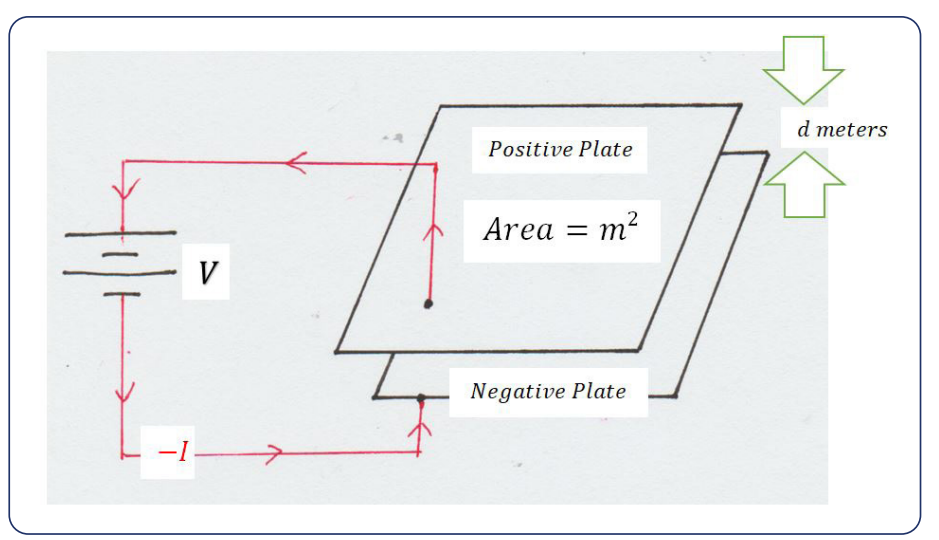

Figure 8a

In Figure 8a, there are two metal plates with a small distance $d$ meters between them. A battery with a voltage $\mathrm{V}$ is connected to the two plates. The negative end of the battery is connected to the negative plate and the positive plate is connected to the positive end of the battery. As soon as the battery is connected to the two plates, the free valence electrons (-e) from the negative pole of the battery start flowing as a negative electric current -I into the Negative Plate as much as possible. The free valence electrons (-e) on the Positive Plate are repelled towards the positive pole of the voltage source $\mathrm{V}$.

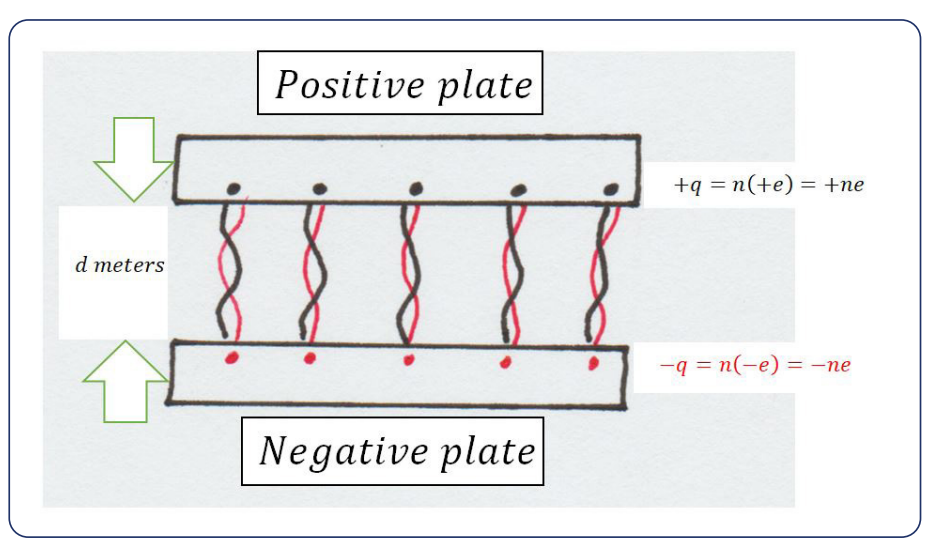

Figure 8b
The free valence electrons (-e) gathered on the surface of the Negative Plate and owing the small distance of $d$ meters repel the free valence electrons (-e) in the Positive Plate towards the positive pole of the battery or voltage source $\mathrm{V}$ and we have exposed protons (+e) also called 'holes' in electrical engineering due to lack of some electrons in the Positive Plate facing the free valence electrons on the Negative Plate of the Capacitor.

When the Capacitor is fully charged:

From the Negative Plate the negative electric field lines with a negative torque $(-\tau)$ head for the Positive Plate.

From the Positive Plate the positive electric field 1 ines $w$ ith a positive torque $(+\tau)$ head for the Negative Plate.

In Figure $8 b$, the positive electric field lines from the Positive Plate head for the Negative Plate and negative electric field lines from the Negative Plate head for the Positive Plate and entwine with each other and appear pretty much like the electric field lines from the two poles of a magnet.

The charged Capacitor serves as a reservoir of electric energy until some action is taken to use this charge.

Capacitor charge $\mathrm{q}=\mathrm{CV}$ where $\mathrm{C}$ is for capacitance and $\mathrm{V}$ is for voltage of source.

From Gauss' law $\varepsilon_{0} \oint \mathrm{E}$. dA $=\mathrm{q}$ or $\varepsilon_{\mathrm{o}} \mathrm{E} \oint \mathrm{dA}=\mathrm{q}$

But $\oint \mathrm{dA}=\mathrm{A}$ the surface area of the plate and $\varepsilon_{0} \quad$ is the permittivity constant.

So $\varepsilon_{0} \mathrm{EA}=\mathrm{q}$ coulombs.

Voltage $\mathrm{V}=-\int_{0}^{\mathrm{d}} \mathrm{E}$. ds and as $\mathrm{E}$ is a constant in this situation

$\mathrm{V}=-\mathrm{E} \int_{0}^{\mathrm{d}} \mathrm{ds}$, as $\int_{0}^{\mathrm{d}} \mathrm{ds}=\mathrm{d}$ meters

$\mathrm{V}=-\mathrm{Ed}$ volts

Capacitance $C=\frac{\mathrm{q}}{\mathrm{V}}=\frac{\varepsilon_{\mathrm{o}} \mathrm{EA}}{-\mathrm{Ed}}=-\varepsilon_{0} \cdot \frac{\mathrm{A}}{\mathrm{d}}$ in Farads.

Some avoid the minus sign.

Capacitance of the Capacitor is directly proportional to the area of the two capacitor plates and is inversely proportional to the distance between the two plates. A larger voltage source will result in a greater charge on the Capacitor within limits.

Capacitance $\mathrm{C} \infty \mathrm{A}$ and $\mathrm{C} \infty \frac{1}{\mathrm{~d}}$.

Capacitor unit Farad $=\frac{\mathrm{q}}{\mathrm{V}}$ or Coulomb/Volt.

Conclusion: Once charged, the positive and negative forces from the two capacitor plates having positive and negative charges with opposing torques leads to these two forces entwining with each other as in Figure $8 \mathrm{~b}$ and staying put until the Capacitor can discharge when needed. 


\section{DISCUSSION}

'Gill's electronic theory of magnetism 1964' is based on the structure of the atom and the magnetic force is a combination of the exposed inner electron (-e) based north magnetic pole $\mathrm{N}$ and the exposed proton $(+e)$ based south magnetic pole $\mathrm{S}$ and not a single force as previously stated.

This magnetic force also has a negative torque $(-\tau)$ passed on from the negative exposed inner electrons (-e) with a negative torque $(-\tau)$ which is the north magnetic pole $\mathrm{N}$ combined with a positive torque $(+\tau)$ passed on from the positive exposed protons $(+e)$ with a positive torque $(+\tau)$ which is the south magnetic pole $S$ as these forces are coming out of the two ends of a magnet to entwine.

If the magnetic force $\mathrm{B}$ approaches another magnet, its two components will split and head for the opposite magnetic poles of the second magnet as explained in the 'Message from the SUN' article by author in ARJPS 2017.

While addressing the magnetic force or the electrical force, we are dealing with a combination of the electron (-e) based negative force and the proton $(+\mathrm{e})$ based positive force.

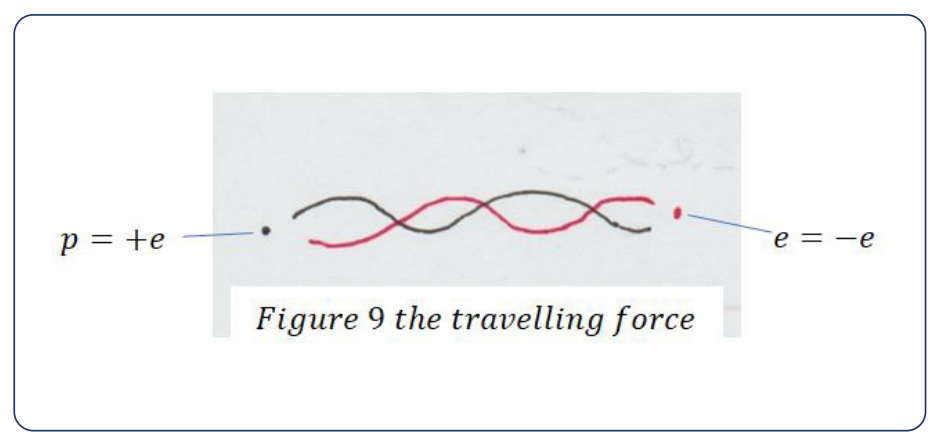

As shown in Figure 9, the travelling force is a combination of the electron (-e) based negative force and the proton $(+\mathrm{e})$ based positive force as they entwine while heading for each other.

Faraday's law of induction has been explained with the help of Gill's electronic theory of magnetism 1964 applied to Faraday's iron ring experiment by the author in ARJPS 2017.

A modified version of Gauss' law has been presented which further explains the similarity between the magnetic force combination and the positive and negative electrical force combination.

The Capacitor is discussed and how the negative and positive forces from the two plates entwine until the reservoir charge is discharged. It is once again stressed that we should present the direct electric current flow as a negative electron (-e) flow.

The working of a Cyclotron has been presented and how a moving positive ion with a velocity $\mathrm{v}$ is repelled by the positive torque $(+\tau)$ of $\mathrm{B}$ in a circular manner is explained. No cross products of Henrik Lorentz
1893 required.

The Electric Motor rotates because of the negative torque (- $\tau$ ) from the negative force component of the magnetic force B of the Stator repelling the moving electrons perpendicularly $\perp$ to the direct electric current in the coil.

The (Coil effect) ${ }^{\mathrm{n}}$ and how to put it to u se to multiply the mild electrical energy which is everywhere is presented.

Applying Gill's electronic theory of magnetism 1964, dot products are sufficient and no need of cross products of Henrik Lorentz 1893.

Gill's electronic theory of magnetism 1964 leads to easy conversion between the force in Newtons and the Tesla force. It helps resolve the asymmetry issue which caused A. Einstein to enunciate the Special Theory of Relativity in 1905.

\section{CONCLUSION}

Electrical Engineering will be better served if Gill's electronic theory of magnetism 1964 replaces Maxwell's dipole theory of magnetism 1873 as dot product equations are enough without any need of Henrik Lorentz's cross product equations 1893.

The asymmetry issue is resolved and this had caused A. Einstein to enunciate the Special Theory of Relativity in 1905.

Renewable energy with the application of the (Coil effect $)^{\mathrm{n}}$ could go a long way in decreasing the dependance on oil.

Suggested power-point videos of articles by author:

1. Faraday's iron ring experiment (1831) explained with Gill's electronic theory of magnetism (1964) by author ARJPS 2017. https://drive.google.com/file/d/1X18SbpVo9Ocg-qeiMPWI5Owqa1e_8ei/view?usp=sharing

2. Message from the SUN with Gill's electronic theory of magnetism (1964) by author ARJPS 2017.https://rive.google.com/file/ d/1APZtS09eGkRtguhCx02WLc-HS0jyd35s/view?usp=sharing

3. Meissner effect re-explained with Gill's electronic theory of magnetism (1964) by author ARJPS 2018.https://drive. google.com/file/d/1Q8pNRrTHZ4hUjuQ53Uj9cJHyzIJ9Dyuk/ view? usp=sharing

4. Bullet train physics with Gill's electronic theory of magnetism 1964 by author ARJPS 2018.https://drive.google.com/file/d/1en8c 3KQVx8JDvR2HVVSPvMnN17RhrJ1Y/view?usp=sharing

5. How Objects Fall by author in ARJPS 2019 (improved version). https://drive.google.com/file/d/1AC4aNYs5sIGgpL0M0TMxjjf4 Tl6kvCRs/view?usp=sharing 


\section{REFERENCES:}

1. Coulomb's law by Charles Augustin de Coulomb1784.

2. A Treatise on Electricity and Magnetism by James ClerkMaxwell.

3. Ampère's circuital law by Andre Marie Ampere1823.

4. Lorentz force by Hendrik Lorentz1893.

On the electrodynamics of moving bodies By A. EINSTEIN June 1905.

5. Tesla unit by Nikola Tesla.

6. Khan academy of Salman Khan.

7. Scientific tutorials of Jason Gibson.

\section{FOOT NOTES:}

1. Previously called 'Thakar's electronic theory of magnetism 1964' under my pen name Thakar.

2. Already presented in detail in an article in ARJPS in 2017.

3. Already done in a previous 'Bullet Train Physics' article in ARJPS in 2018.

4. From Wikipedia. Suggested reading 'A Treatise on Electricity and magnetism by James Clerk Maxwell 1851'.

5. Refer Meissner effect re-explained with Gill's electronic theory of magnetism 1964 by author in ARJPS 2018.

6. Jason Gibson's physics tutorials help but the author differs.

7. For applications see Message from the SUN with Gill's electronic theory of magnetism 1964 by author in ARJPS 2017.

Citation: Avtar Singh Gill "Electrical Engineering 2020 with Gill's electronic theory of magnetism 1964". American Research Journal of Physics, Vol 7, no. 1, 2021, pp. 1-12.

Copyright $\odot 2021$ Avtar Singh Gill, This is an open access article distributed under the Creative Commons Attribution License, which permits unrestricted use, distribution, and reproduction in any medium, provided the original work is properly cited. 